\title{
Reactivating the phototransduction cascade by universally applicable gene therapy preserves retinal function in Rod-Cone dystrophy
}

\section{Cardillia-Joe Simon}

Institut de la Vision

Hanen Khabou

Vision Institute, Sorbonne Univ.

Marion Finzi

Institut de la Vision

Antoine Chaffiol

Sorbonne University

\section{Kate Grieve}

Institut de la Vision

Nat Norberg

CHNO des Quinze-Vingts, INSERM-DGOS CIC

\section{Anais Grimaud}

CHNO des Quinze-Vingts, INSERM-DGOS CIC

Dennis Eickelbeck

The Francis Crick Institute https://orcid.org/0000-0002-8494-6543

Marco Rucli

Institut de la Vision

Mélissa Desrosiers

Institut de la Vision

\section{Serge Sancho}

CHNO des Quinze-Vingts, INSERM-DGOS CIC 1423,

\section{Vera Bonilha}

The Cleveland Clinic https://orcid.org/0000-0002-6166-5124

\section{Saddek Mohand-Said}

Clinical Investigation Center, Quinze-Vingts National Eye Hospital

Jens Duebel

Michel paques

Quinze-Vingts hospital

\section{Serge Picaud}

Sorbonne University https://orcid.org/0000-0002-0548-5145 
José-Alain Sahel

Sorbonne University

Isabelle Audo

Sorbonne Universités UPMC Univ Paris 06 UMR_S 968 Institut de la Vision https://orcid.org/00000003-0698-5309

\section{Stefan Herlitze}

Ruhr University Bochum

Deniz Dalkara ( $\nabla$ deniz.dalkara@gmail.com)

Sorbonne Université, Institut de la Vision, INSERM, CNRS https://orcid.org/0000-0003-4112-9321

\section{Article}

Keywords:

Posted Date: January 17th, 2022

DOI: https://doi.org/10.21203/rs.3.rs-1189099/v1

License: (c) (i) This work is licensed under a Creative Commons Attribution 4.0 International License. Read Full License 


\section{Abstract}

Rod-cone dystrophy (RCD), also known as retinitis pigmentosa, is a group of inherited disorders characterized by rod degeneration followed by degeneration of peripheral cones, leaving patients with tunnel vision in mid stages and blindness in the latest stages. Previous studies expressed microbial chloride pumps, in degenerating cone photoreceptors to restore these cells' activity and prolong vision. Microbial opsins lack intracellular signal amplification, requiring high light intensities. To develop a more sensitive strategy; we examined the phototransduction cascade in degenerating cones, in two RCD mouse models. We found that opsin and arrestin expression is maintained in the soma during outer segment degeneration. We thus hypothesized that cone reactivation based on cone opsin signaling may be feasible by expressing a target channel activated by $\mathrm{G}$ proteins recruited by cone opsin. Adeno-associated viral (AAV) mediated expression of $\mathrm{G}$ protein coupled inwardly rectifying $\mathrm{K}$ (GIRK) channel provided improvements in visual function in two RCD mouse models with mutations in two distinct genes. Importantly, we confirmed cone opsin and cone arrestin expression in cones of late-stage RCD patients, validating the rationale of GIRK-mediated gene therapy in humans. We propose GIRK channel expression in cones as a new approach to maintain high acuity, high sensitivity, color vision in RCD independently of the underlying mutation.

\section{Introduction}

Inherited retinal degenerations are mostly due to mutations in photoreceptor or retinal pigmented epithelial (RPE) cells. Among these retinal degenerations, rod-cone dystrophy (RCD) represents the largest category characterized by the degeneration of the rod photoreceptors first, followed by cone photoreceptor outer segments' disappearance leading to blindness (1). RCD are highly genetically heterogeneous. More than 60 different genes expressed in rods or the retinal pigment epithelium are involved (2). The first gene that has been associated to RCD is the rhodopsin gene $R H O$ that accounts for $25 \%$ of RCD autosomal dominant cases. Many other genes have also been associated with RCD including genes encoding the cGMP-phosphodiesterase (PDE) $\beta$ or a subunit and the cyclic GMP-gated channel protein a or $\beta$ subunit. Although there are numerous genes involved, the resulting RCD phenotype follows a similar phenotype in affected patients (3). This phenotype starts with progressive rod degeneration, causing night blindness. However, cones remain until late stages of the disease; particularly in the foveal region responsible for high acuity, which leads to tunnel vision (4). At later disease stages, these cones lose their outer segment structures leading to complete blindness. Afterwards the complete loss of the cone soma and pedicle occurs in part of the patients (4). Preserving the remaining high-acuity central vision in patients has been a major goal of recent gene therapy studies (5-9).

Indeed, the first successful clinical trials of gene therapy have focused on gene addition, where a wildtype cDNA copy is brought by a vector to supplement the defective gene. This approach is reserved to patients in early stages of the disease, having intact photoreceptors where the causal mutation is known and leads to a loss of function of the resulting protein. Gene addition is not applicable to the majority of retinal degeneration patients who are diagnosed too late or suffer from dominant negative mutations 
(10). As of today, in $30 \%$ of cases, the causative mutation is not elucidated (11) or the rod photoreceptors bearing the most frequent mutations are already lost. For these reasons, mutation-independent gene therapies that can be applied beyond the loss of rods must be developed to treat a larger number of patients without knowledge of the mutant gene.

Optogenetics has recently emerged as a mutation-independent therapeutic strategy to restore vision in blind patients (12). The ectopic expression of microbial opsins like channelrhodopsin in retinal ganglion cells, or the pump halorhodopsin in remaining cone photoreceptor cell bodies, modulates the membrane potential and induces a light-dependent depolarization or hyperpolarization, respectively (13-20). This strategy which has been successfully implemented in clinical stages is limited by the high light intensity requirements of microbial opsins, thereby requiring the need for signal-amplifying goggles together with the gene therapy $(16,20)$. Indeed, light sensitivity of these directly light gated channels and pumps originating from single cell microorganisms is much lower than those of mammalian rhodopsins or cone opsins, which activate $\mathrm{G}$ protein-coupled cascades such as the phototransduction cascade present in healthy mammalian cones (Fig. 1A). One of the possible ways to go beyond this limitation of microbial opsins is to use animal opsins, which are $G$ protein-coupled (21-26). In addition to these findings reported in the retina, a previous study has shown that light activation of animal cone opsins can stimulate the $\mathrm{G}_{\mathrm{i} / \mathrm{o}}$ signaling pathways in human kidney cells and in brain neurons (27). Membrane potential modulation was possible in these cells by ectopically expressed cone opsins thanks to the intrinsic presence or ectopic expression of $\mathrm{G}$ protein-gated inwardly rectifying potassium channels (GIRK) which acts as a target molecule to the $G$ proteins recruited by the opsins $(21-26)$. Indeed, GIRK channels are activated in excitable cells by GPCRs coupling to the $\mathrm{G}_{\mathrm{i} / \mathrm{o}}$ pathway. After their activation by the $\beta y$ subunit of a $G_{i / o}$ protein, potassium ions flow out of the cell, thus, hyperpolarizing the membrane (Fig. 1B). It has therefore been possible to use vertebrate cone opsins for repetitive $G_{i / o}$ activation upon illumination in vivo in the anxiety circuitry, and the combination of cone opsins with GIRK has proven more efficient than microbial opsins at low light intensities. Indeed, for vertebrate opsins light-induced GIRK currents were largest around $0.02 \mathrm{~mW} / \mathrm{mm}^{2}$ whereas microbial opsins were maximally activated at $2 \mathrm{~mW} / \mathrm{mm}^{2}$ (27). These findings suggest, it might be possible to use cone opsin and GIRK to enhance light sensitivity of degenerating cones with diminished outer segments.

To test the feasibility of GIRK mediated activation of degenerating cones, we first investigated the state of the endogenous phototransduction cascade through the progression of the disease. We found that endogenous cone opsins are still expressed on the membrane of cone cell bodies in both mouse models and in several RCD patients' maculae post-mortem, suggesting the possibility of linking their activity to GIRK channels even in absence of transducin and phosphodiesterase (Fig. 1), which are downregulated in later stages of degeneration (27). Upon AAV-mediated GIRK2 gene transfer, significant visual improvements are seen in two mouse models of RCD with different underlying mutations. Our results point towards enhanced light-sensitivity in cone photoreceptors of RCD mouse models during degeneration of cone outer segments. This is the first time a light insensitive mammalian ion channel has been linked to intrinsic opsins offering new avenues in vision restoration, that can be implemented to 
increase light sensitivity even before complete outer segment degeneration. Since our system makes use of intrinsic opsins expressed in degenerating cones, it would also enable for the first time, color vision restoration which has not been feasible in previous studies of optogenetics. Our preliminary retrospective clinical study shows a significant proportion of retinitis pigmentosa patients who have very reduced or lost light perception still have a detectable ONL filled with diminished outer-segment cone photoreceptor cells. Since cones in the macular region provide up to $50 \%$ of the input to the visual cortex (29), targeting these cones with our new approach promises vision restoration respecting the normal retinotopy and high visual acuity characteristic of this area.

\section{Results}

\section{Changes in cone phototransduction cascade proteins in the rd10 mouse model}

To provide an understanding of the changes in the phototransduction cascade in RCD, retinal samples were collected from homozygous $\mathrm{rd} 10$ mice between post-natal days 21 and 150 . Cross sections were stained for cone phototransduction proteins in comparison with wild-type retinal cross sections. Target proteins for immunohistochemistry were mouse medium-wave-sensitive cone opsin 1 (Opn1mw), transducin (Gnat2), cone phosphodiesterase (Pde6c) and cone arrestin (Arr3) of the phototransduction cascade across different ages (Supp Fig. 1 and Fig. 2). At day 150, we found that only the cone opsin and cone arrestin proteins were still expressed at detectable levels and localized to the cone cell body while Gnat2 and Pde6c were no longer detectable by immunohistochemistry (Fig. 2), To get more insight into the decline of cone function with respect to the declining expression of cone phototransduction transcripts, we further examined the phototransduction cascade gene expression in the wild-type and rd10/rd10 mice at postnatal day 150 by quantitative RT-PCR (Supp Fig. 2) in rd10 mice compared to wt BL6J mice. Gnat1 was used as a control as there are no longer rods. As expected, no expression was detected in the rd10 retina. Opn1sw, Opn1mw, Arr3, Cnga3, Pde6c, and Gnat2 were quantified at P150. Opn1sw, Opn1 $\mathrm{mw}$ and Arr3 transcript levels in the $r d 10$ retinas were significantly lower than those in the wild-type retinas at P150 (Supp Fig. 2) as previously reported for another mouse model of RCD (31). An overall decrease in the level of membrane bound opsin is coherent with the loss of the outer segment which contains most of the membrane surface in normal cones. In absence of outer segment, there is less transcript, yet, translation of opsin and arrestin mRNA is still ensured at levels detectable by histology. On the contrary, Cnga3 and Pde6c transcript levels were not significantly different than wildtype levels although $P D E 6 c$ protein is not detected by immunohistochemistry at P150. This result can be attributed to a dysfunction in the translation pathway of $P d e 6 \mathrm{cmRNA}$ into proteins at later stages of cone degeneration. Finally, Gnat1 (rod photoreceptor G-protein) transcript level was null as rod decay is compete at P150.

\section{GIRK2 expression in cones increases photopic light responses in degenerating rd10 retinas}


Based on immunohistochemistry and previous findings with cone opsins expressed in neurons, we reasoned that delivering an AAV vector encoding a GIRK channel can enhance cone cells' response to light by endogenous cone opsins recruiting a G-protein (Fig. 1B). GIRK2 channels are capable of forming functional homotetramers (30). We thus chose a truncated rat GIRK2 as a target channel to be expressed in cones. We used AAV8 as this capsid is known to have high transduction capacity towards mouse photoreceptor cells and we combined it with the promoter PR1.7 which is a strong cone specific promoter $(19,32)$. We performed bilateral subretinal injections on rd10 mice at P15 as detailed in Table 1.

Table 1

Experimental groups, viral vector doses and the injection volume.

\section{Injection Table}

Eyes Viral vector injected

Right PBS-pluronic $0.001 \%$

Left $\quad$ AAV8-PR1.7-GIRK2-GFP
Viral vector dose

Volume injected

$1 \mu \mathrm{L}$ for all conditions

\section{Longevity of GIRK2 mediated light responses in rd10 retinas}

$5.10^{8}$ AAV particles

To evaluate the longevity of functional benefits obtained by ectopic GIRK2 expression in cones, we performed photopic ERG recordings at different time points after injections. We recorded photopic ERGs under two conditions: (i) photopic with white light flashes applied every second during 60 seconds at increasing light intensities (Fig. $3 \mathrm{~A}$ ) and (ii) $10 \mathrm{~Hz}$ flicker stimulation with repetitive flashes at the same intensity for 60 seconds (Fig. 3B). Data were collected on a weekly basis until P50 and then every 10 to 20 days until 20 weeks of age. Treated eyes showed a significant increase in ERG amplitudes compared to control eyes until P120 (Fig. 3C). At 5 months of age, the difference between treated and untreated eyes was no longer detectable under these conditions. This is due to the detection threshold of the ERG that requires a minimum number of active cones in order to have a recordable response to light stimuli. As a more sensitive readout, we evaluated behavioral responses of treated mice by optokinetic test (OKT). Significant differences in visual acuity of treated and untreated eyes was visible even at the latest time point (5 months old) (Fig. 3D). Nevertheless, a gradual decline in both ERG amplitudes and in OKT responses was observed, with or without treatment. This decline can be attributed to decrease in cone numbers over time as GIRK2 treatment does not increase survival of cones (Supp Figure 3B).

\section{GIRK2-mediated vision restoration in a rhodopsin mutant RCD mouse mode}

To test whether GIRK2 can be useful to increase cone-mediated light responses in a mutationindependent manner, we tested our approach in another RCD mouse model. mRho ${ }^{+/-}$huRhoP3475 ${ }^{+/+}$ (huP3475 ${ }^{+/-}$) carrying a knock-in P347S mutant human rhodopsin was used to perform the identical set 
of experiments (33). First, we analyzed the phototransduction cascade proteins interacting with cone opsin at different time points (Fig. 4 and Supp Figure 4). Similarly, to rd10 model cone opsin and cone arrestin expression persisted in cone cell bodies at P150 but the phosphodiesterase and the transducin proteins were undetectable at this time point.

Next, we injected mice at P15 with the same AAV vector encoding for GIRK2 fused with GFP (Table1) and recorded ERGs to monitor cone response to light stimuli at various time points (Fig. 5A-B). The amplitude responses of treated eyes were significantly higher than that of control eyes until P100 (Fig. 5C). This confirmed that our approach is a mutation-independent therapy that preserves vision at late stages regardless of the causal RCD mutation. Moreover, we recorded similar ameliorations in flicker ERG responses in this mouse model (data not shown). For the same reasons as $r d 10$ mice, i.e. progressive loss of cones, no significant difference in light responses was observed in treated eyes compared to control eyes at 5 months. However, treated eyes showed an improved visual acuity compared to control eyes until P150 (Fig. 5D). Similarly, to rd10 mice, a gradual decline in ERG amplitudes and in behavioral responses can be observed in treated eyes as well as control eyes but treated eyes displayed improved function compared to controls. The decline in number of cones (Supp Figure 3B) is again consistent with the fact that our approach does not stop the degeneration but allows for enhanced light sensitivity of cones through GIRK2.

\section{GIRK2-mediated vision restoration: feasibility in RP patients}

Is GIRK gene therapy translatable to RCD patients? To check the applicability of this approach at the cellular level in the human retina, we used post-mortem retinal specimen to analyze phototransduction cascade proteins in the macular region in 4 RP patients aged between 73 and 92 years old (see Supplementary Table 1) who were in intermediate and advanced stages of the disease (Postmortem eyes obtained from the Cole Eye Institute Eye Tissue Repository through the Foundation Fighting Blindness (FFB) Eye Donor Program (Columbia, MD)). We also analyzed the expression of phototransduction cascade proteins in a healthy control retina from a 91-year-old patient (tissue was obtained from the Surgery School of Paris). Importantly, we found that similar to RCD mouse models, cone opsin and cone arrestin remain in cone cell bodies of RP patients (Fig. 6). Indeed, three of four donors (Donor 2 to 4 ) had cones with diminished outer segments. In all 3 patients, cones were found co-expressing opsin and arrestin (Fig. 6B-D). The donor 5, who was blind, had only a few sparse cones remaining (Fig. 6E). Altogether these data confirm the applicability of GIRK-mediated gene therapy in human cones. Our short phototransduction cascade strategy can potentially reactivate cone function in RCD patients. The activation of remaining cone opsin by a light stimulus would therefore trigger the short GIRK2-mediated phototransduction cascade and lead to enhanced light sensitivity in RP patients.

To address more closely the question of applicability in patients, we investigated the proportion of potential eligible patients in our RCD cohort from the center of rare disease of the Quinze-Vingts Eye Hospital (Paris). We sorted out patients based on two criteria: (i) visual acuity $<2 / 10$ with low to no light perception and (ii) presence of a detectable ONL filled with cone photoreceptor cells displaying shortened 
or absent outer-segments (referred to as 'dormant cones') in optical coherence tomography (OCT) bscans (Spectralis, Heidelberg Engineering, Germany). As a preliminary starting point, we screened a database of 350 eyes with genetically confirmed Retinitis Pigmentosa (RP) diagnosis and we sorted eyes having a visual acuity below 2/10 (Figure 7A). We focused on those patients because our approach using GIRK2 would increase light sensitivity in degenerating cone photoreceptor cells and allow the patient to regain light perception and high acuity vision in the fovea. Based on these criteria, we eliminated 235 eyes, and we focused on 115 eyes with very reduced or lost light perception (Fig. 7A). Then, we eliminated eyes with no discernible ONL by examining the fine structure of the foveal region containing the cone cells last to degenerate in RCD (also referred to in the literature as remnant cones or dysflective cones(34)), targets of our gene therapy approach. Among the 115 eyes with low to no light perception, 29 eyes had a distinguishable outer nuclear layer (ONL) composed of cones with shortened or absent outersegments (Fig. 7A-E). This indicates that roughly one quarter of RP patients with low to no light perception can be eligible for GIRK2 therapy. In order to deeply characterize the dormant cone phenotype, and examine the inner segment structures overlying the diminished outer segments, we analyzed the cone cells of a patient with a very high-quality OCT scan, using adaptive optics scanning laser ophthalmoscopy (AOSLO) (Physical Sciences Inc., Andover MA, USA)(Fig. 7C). Intact cones were captured in the confocal channel and intact inner segments in the split detection channel. Refractive changes in the inner and outer segments of the foveal cones suggested it is possible to identify the presence of light insensitive 'dormant' cone population using this technique (Fig. 7G). The combination of the abovementioned imaging techniques along with more recently described indicators of cone cell function (such as fundus autofluorescence imaging(31)), makes it feasible to select patient populations that would most benefit from GIRK2 gene therapy.

\section{Discussion}

Previous work had shown the long-term presence of degenerating cones in RP patients and mouse models (17-19). These cones are often referred to as 'dormant cones' once their outer segment is significantly diminished. Such cones retaining the nucleus and inner segment and perhaps remnants of outer segments are also referred to as dysflective cones or more generally as remnant cones (34). These cones may be the cells most likely to respond to therapies designed to prevent cell death and restore visual function. Cone photoreceptor reactivation studies using optogenetics showed the feasibility of restoring vision in high light conditions using microbial opsin-based optogenetics (17-19). However, the high light requirements and the potential immunogenicity of using an opsin from prokaryotic origin are inherent disadvantages of such an approach. An interesting alternative is the use of mammalian opsins for vision restoration, however all work in this field has so far been focused on inner retinal neurons (23, $25,35-37)$. After exploring the phototransduction cascade in two RCD mouse models, we propose, a new gene therapy strategy based on remaining endogenous cone opsins (Fig. 1). Indeed, we revealed that cone opsin and cone arrestin remain expressed in cone cell bodies at late disease stages both in mouse models of RP and in human RP patients. Based on this information, it is plausible to insert a channel acting via $\mathrm{G}_{\mathrm{i} / \mathrm{o}}$ proteins recruited by the activation of the remaining opsin and thereby creating an 
alternative 'short phototransduction cascade' within the cone photoreceptor (Fig. 1B). Such phototransduction cascade provides increased light responses as long as endogenous cone opsins are still present in viable cones. We propose that in early stages, while transducin is still present, the activation of the opsin by a light stimulus recruits the a subunit of transducin leaving the $\beta y$ subunits available for activation of GIRK2 channels, generating additional hyperpolarization (Fig. 1B). Alternatively, or additionally in later stages when transducin is no longer present, the opsin can recruit other $\mathrm{G}$ proteins present in degenerating cones targeting GIRK2 channels, subsequently allowing the efflux of potassium ions at the resting membrane potential of degenerating cones (17). $\mathrm{K}^{+}$efflux via GIRK2 channel hyperpolarizes cone photoreceptors in response to light modulating glutamate release and light responses in two RCD mouse models by ERG and OKT. Since remaining opsin in cone cell bodies is still functional within its regular spectrum, the insertion of GIRK2 in all cones with PR1.7 promoter leads to light responses following the spectral properties of each of the opsins, therefore, allowing the preservation of color vision. We thus anticipate that our approach will provide, for the first time, colorvision restoration with both high acuity and sensitivity.

A clear advantage of microbial opsins is their robustness and millisecond scale kinetics $(20,38)$. For systems using other opsins, it should be considered that in order to respond to another light stimulus, the cascade has to be deactivated to recover light sensitivity. In absence of this, cones may stay hyperpolarized after GIRK2 channel activation limiting their ability to modulate synaptic transmission at a movie rate compatible with motion vision. In our case, signal termination in the cones was made possible thanks to the cone arrestin that is still maintained at advanced stages of the disease in both RCD models and patient retinas. This is readily visible in the $10 \mathrm{~Hz}$-flicker ERG traces showing responses of the retina during repetitive light stimuli and also by the improved optokinetic reflex of treated mice.

Our observations of 'dormant cones', i.e. degenerating cones with diminished outer segments and light sensitivity, in human RP retinas is consistent with previous reports $(4,17,39-41)$. Li et al. (4) reported somata with very short or absent OS reactive for cone opsins, recoverin, and transducin- $\alpha$ cones in several RCD patients while Lin et al. (41) also found cones with abnormal, diminished OS that were positive for recoverin immunostaining in one RCD patient. Busskamp et al. (17) showed the presence of such cones using OCT in RCD patients. We show here the presence of this type of cones in the maculae of 3 of 4 RP patients studied. Importantly, we found co-expression of cone opsins and cone arrestin in the same cells, which supports our GIRK2 gene therapy approach as these two phototransduction cascade proteins are required for our cone reactivation strategy using GIRK2. Last, the fact that incorporation of GIRK2 enhances existing light responses in cones even prior to complete outer segment loss offers the possibility to implement this gene therapy in mid stages of the disease.

Based on our investigation of the proportion of potential eligible patients in our RCD cohort from the Quinze-Vingts hospital we found that roughly one quarter of RP patients with low to no light perception can be eligible for GIRK2 therapy to restore light sensitivity in their dormant cone population. Examination of the inner and outer segment structures in RCD patients using AO imaging revealed refractive changes in the inner and outer segments of the foveal cones suggesting it is possible to distinguish between 
cones with diminished outer segments, inner segments and light insensitive 'dormant' cone populations using this technique. The combination of the above-mentioned imaging techniques along with more recently described indicators of cone cell function (such as fundus autofluorescence imaging (42)) can be used to select patient populations for this type of gene therapy.

Despite functional improvements in GIRK2 expressing cones, our treatment does not stop the degeneration of cones. However, retinal degeneration in mice is much faster than in humans, thus a few months of therapeutic efficiency in mice may be equal to several years in humans. We recorded a decrease in the response of treated cones to light stimuli, which was consistent with decrease in cone numbers and the fact that we did not transduce all cones due to subretinal injection further limiting the beneficial effect. AAV vectors showing better lateral spread can be used to increase transduced cone numbers beyond the bleb (19). In order to increase the therapeutic window, neurotrophic factors can be implemented alongside our approach. Indeed, AAV-mediated secretion of neurotrophic factors such as the rod-derived cone viability factor (RdCVF) have been shown to delay cone cell death and may be combined with GIRK2-mediated sensitization (5).

Guanine nucleotide-binding proteins act as molecular switches inside a multitude of cells (43). They have a seven-transmembrane domain receptor, and their activity is regulated by factors that control their ability to bind and hydrolyze GTP to GDP. The most commonly identified G proteins in the retina are heterotrimeric and are composed of three subunits $(a, \beta, \gamma)$. They are activated by light sensitive $G$ proteincoupled receptors (GPCR) such as rhodopsin or cone opsin and transmit the message by activating other proteins in the cascade. In photoreceptors (PRs), the $G$ protein that activates the cascade (transducin) belongs to the $G_{i / o}$ family inhibiting the production of cyclic guanosine monophosphate (cGMP) from GTP and therefore inducing hyperpolarization of photoreceptor membrane and a subsequent decrease of glutamate release (44). Recently, the expression of a medium wavelength-sensitive cone opsin in the retinal ganglion cells has been shown to restore high light sensitive vision with adaptation (21). Even though the $\mathrm{G}$ protein coupled mechanism used by cone opsin in retinal ganglion cells remains unknown at this stage, such results further show the potential of vertebrate cone opsins in vision restoration. They also suggest that cone opsins are not specific to a single type of $\mathrm{G}$ protein with the ability to activate target channels in different neurons (45). Various ion channels can be activated via a $\mathrm{G}$ protein starting with the light-stimulated opsin. These findings altogether lead us to believe that our combined approach with GIRK2 or a similar dual approach with a target channel and opsin could be implemented in other subtypes of neurons broadening the field of applications towards other targets (27).

\section{Materials And Methods}

\section{Animals}

$\mathrm{C} 57 \mathrm{BL} / 6 \mathrm{j}^{\text {rd10/rd10 }}(\mathrm{rd10})$ mice were used in these experiments. They carry a mutation in Pde6b gene expressed by rods, leading to a dysfunctional phototransduction cascade and a rod-cone dystrophy. The second model used is the huP3475 ${ }^{+/-}$mouse. The homozygous strand of this mouse presents a KO of 
mouse rhodopsin Rho gene and a KI of a mutated human rhodopsin RHO gene with a P347S mutation (45). Homozygous males were crossed with C57BL/6j (wild type) females to obtain heterozygous mice. These mice have a similar phenotype as the $r d 10$ mice, but the degeneration rate is slower.

\section{AAV Injections}

Mice were first anesthetised with isofluorane inhalation (5\% induction and $2 \%$ sustain). Eyes were dilated with $8 \%$ Neosynephrine (Neosynephrine Faure 10\%, Europhta) and 42\% Mydriaticum (Mydriaticum 0,5\%, Thea) diluted in $0.9 \% \mathrm{NaCl}$. Then eyes were protected with Lubrithal eye gel (VetXX).

A total volume of $1 \mu \mathrm{l}$ of vector solution was injected subretinally with a Hamilton syringe, in the dorsaltemporal area of the retina. Different mice were used for ERG recordings $(n=6)$ and OKT recordings $(n=6)$. Exclusion criteria for subretinal injections were haemorrhages, absence of retinal detachment and lens damage. Fradexam, an ophthalmic ointment, was applied after injection.

\section{Mouse eye fundus examination}

One week after subretinal injection, mice were anesthetised by isofluorane inhalation ( $5 \%$ induction and $2 \%$ sustain). Eyes were dilated with $8 \%$ Neosynephrine (Neosynephrine Faure 10\%, Europhta) and $42 \%$ Mydriaticum (Mydriaticum 0,5\%, Thea) diluted in $0.9 \% \mathrm{NaCl}$. Then protected with Lubrithal eye gel (VetXX). Fundus imaging was performed with a fundus camera (Micron III; Phoenix Research Lab) equipped with specific filters to monitor GFP expression in live anesthetized mice.

\section{Electroretinography (ERG) recordings}

Prior to functional tests, OCT imaging was performed to check for spontaneous retinal separations described for $r d 10$ mice (46) and mice showing spontaneous detachments were not recorded. To evaluate retinal function, full-field electroretinograms (ERG) were recorded (Espion E2 ERG system; Diagnosys $\AA$ ). Several tests were performed at different time points after injections of the viral vectors. Mice were anesthetised with intraperitoneal injections of $0.2 \mathrm{~mL} / 20 \mathrm{~g}$ ketamine (Ketamine 500 , Vibrac France) and xylazine (Xylazine $2 \%$, Rompun) diluted in $0.9 \% \mathrm{NaCl}$. Eyes were dilated with Neosynephrine (Neosynephrine Faure 10\%, Europhta) and Mydriaticum (Mydriaticum 0,5\%, Théa) diluted in $0.9 \% \mathrm{NaCl}$. Mice were then placed on a heated pad at $37^{\circ} \mathrm{C}$. The cornea was anesthetized with a drop of Oxybuprocaïne $(1.6 \mathrm{mg} / 0.4 \mathrm{~mL}$, Théa). Eyes were protected with Lubrithal eye gel before putting electrodes on the corneal surface of each eye. The reference electrode was inserted under the skin into the forehead and a ground electrode under the skin in the back.

ERG recordings were done under photopic conditions: (i) single flashes with increased intensities, which reflects cone-driven light responses $-6 \mathrm{~ms}$ light flashes were applied every second during 60 seconds at increasing light intensities $\left(0,1 / 1 / 10 / 50 \mathrm{~cd} . \mathrm{s} / \mathrm{m}^{2}\right)$ with a background illumination of $20 \mathrm{~cd} / \mathrm{m}^{2}$ after an adaptation of 5 minutes at $20 \mathrm{~cd} . \mathrm{s} / \mathrm{m}^{2}$ - and (ii) flicker responses, which are rapid frequency light stimuli that reflect cone driven responses too (70 flashes at $10 \mathrm{~Hz}$ at $1 \mathrm{~cd} . \mathrm{s} / \mathrm{m}^{2}$ ). 
After ERG recordings, rd10 mice with spontaneous retinal detachment as seen with SD-OCT scan (software: InViVoVue Clinic) were excluded from the analysis(46).

\section{Optokinetic reflex test}

Visual acuity was measured using an optokinetic reflex test scoring the head turning movement of a mouse placed in front of moving bars. Testing was performed using a computer-based machine consisting of four computer monitors arranged in a square to form an optokinetic chamber. A computer program was designed to generate the optokinetic stimuli, consisting of moving alternate black and white stripes. The spatial frequency ranged from 0.03 to $0.6 \mathrm{cyc} / \mathrm{deg}$. The program enabled modulation of stripe width and direction of bar movement.

\section{Immunohistochemistry and confocal imaging}

Animals were sacrificed by $\mathrm{CO}_{2}$ inhalation, and the eyes were enucleated and fixed in $4 \%$ paraformaldehyde-PBS for $1 \mathrm{~h}$ at room temperature. The eyes were then dissected either as eyecups for immunohistochemistry or prepared as flat mounts for cone cell counting. Human retinal samples were obtained from the Foundation Fighting Blindness (USA) and were fixed with a mix of paraformaldehyde and glutaraldehyde. Mouse eyecups and human retinas were cryoprotected with a gradient of PBSSucrose $10 \%$ for $1 \mathrm{~h}$ and then in PBS-Sucrose $30 \%$ overnight, then embedded in Optimal Cutting Temperature (OCT) compound. The $12 \mu \mathrm{m}$ thick cryostat sections were cut and mounted on glass slides. Sections were stained with primary and secondary antibodies (Supplementary Table 2) and DAPI (1:2000). The sections were finally washed in PBS, mounted in Fluoromount Vectashield (Vector Laboratories) and cover-slipped for imaging using a laser-confocal microscopy (Olympus IX81). For flatmount retina staining, the protocol is the same except that we do not cryoprotect the tissue. Images were analyzed using FIJI software.

\section{Cell counts}

Flat mount retinas of $r d 10$ and huP347S ${ }^{+/-}$mice were stained using antibodies against mouse cone arrestin Arr3 (1:10000) and DAPI (1:2000). Double stained cells DAPI+ Arr3+ were counted at different ages. Retinas from 3 animals $(n=6)$ were used for each age. Serial optical sections were obtained to cover the thickness of the entire outer nuclear layer (ONL). Two scanning areas of $211.97 \times 211.97 \mu \mathrm{m}$ were made in each of the four regions in all retinas. Counts of cone cells were performed manually using the FIJI software by the reconstruction of the images ( $z$ stack) covering the entire thickness of the ONL. Average density values of each retina were calculated to obtain the number of cone cells per $\mathrm{mm}^{2}$ at different ages.

\section{Human eye fundus examination}

Volumes of optical coherence tomography (OCT) (Spectralis, Heidelberg Engineering, Germany) b-scans were acquired in patients covering the macular region[K1]. Adaptive optics scanning laser ophthalmoscopy (AOSLO) (Physical Sciences Inc., Andover MA, USA) focused on the photoreceptor layer on $2^{\circ} \times 2^{\circ}$ regions of interest identified from the OCT scans. Adaptive optics corrected for the optical 
aberrations of the eye to achieve diffraction limited cell scale resolution in the patient's retina. The AOSLO was operated in dual channel mode where the confocal channel detects light scattered back on-axis from intact photoreceptors, and the split detection channel detects multiply scattered light emerging from the inner segments. By comparing and combining the appearance of these two channels, intact (inner and outer segment present) and damaged (outer segment absent, or both inner and outer segment absent) cones could be distinguished.

\section{Statistical analyses}

Data was analysed with GraphPad Prism and it was expressed as mean \pm standard error of mean (SEM). Comparisons between values were analysed using unpaired two-tailed non-parametric Mann-Witney's test. A level of $p<0.05$ was considered significant. The labels used were * for $p<0.05$, $* *$ for $p<0.01$, *** for $p<0.001$.

\section{Study Approval}

The human and animal studies described in this work was approved by the appropriate institutional review boards. The animal studies were conducted in accordance with ARVO guidelines and approved by the local ethics committee Charles Darwin. For human studies, written informed consent was received prior to participation.

\section{Declarations}

\section{Author contributions}

CJS performed literature review, conducted experiments including mouse injections and in vivo recordings, cone counting on mouse retinas, histology on huP347S+/- retinas, acquired data, analyzed data and contributed to write the manuscript. MF performed histology on rd10 retinas. HK performed histological analysis of post-mortem human retinas and contributed to RT-PCR experiments, figures, and text to the manuscript. AC performed electrophysiological recordings. KG and NN performed Adaptive Optics experiments on patients and contributed to figures and text to the manuscript. AG and IA analyzed the cohort of patients with a visual acuity below 2/10. DE modelled the GIRK2 channel. MR - a former master student - acquired ERG recordings. MD designed cloning constructs. AG and SS acquired OCT and visual acuity data from RP patients in the Quinze-Vingts hospital (Paris). VLB sampled human retinas. SMS and IA provided and cared for selected study patients. IA and AG critically reviewed the patient study proposal and collected patient data. MP, SP, JAS, JD and SH provided scientific input, and advice on the project and manuscript. DD designed and supervised the experiments and wrote the manuscript.

\section{Acknowledgements}

We thank the Paris Vision Institute core facilities (Animal facility platform, Histology platform, Imaging platform) and particularly Camille Robert of the vector core facility for producing the AAVs. We are 
grateful to Sophie Tran and Neha Mohanpuria, Celina Madjene - former master students for their technical contributions in histology and help with experiments. This work was supported ERC Starting Grants (OPTOGENRET, 309776/JD, REGNETHER 639888/DD), the Centre National de la Recherche Scientifique (CNRS), the Institut National de la Santé et de la Recherche Médicale (INSERM), LabexLifesenses (D.D., J.D.), Sorbonne Université, INSERM, ANR-DFG grant VisionOpsin (D.D.,J.D., S.H.), LabEx LIFESENSES (ANR-10-LABX-65), IHU FOReSIGHT (ANR-18-IAHU-01), Paris Ile-de-France Region under « DIM Thérapie génique » initiative. He2471/23-1 (to S.H.), He2471/21-1 (to S.H.), He2471/18-2 (to S.H.), Priority Program (SPP1926; to S.H.), Project number 122679504-SFB874 project B10 (to S.H.) and Project number $316803389-S F B 1280$ project A07 (to S.H.). National Institutes of Health (EY027750 to VLB), a National Eye Institute P30 Core Grant (P30025585 to the Cleveland Clinic), an unrestricted grant from Research to Prevent Blindness to the Cleveland Clinic Foundation.

\section{References}

1. Buch $\mathrm{H}$ et al. Prevalence and Causes of Visual Impairment and Blindness among 9980 Scandinavian Adults: The Copenhagen City Eye Study. Ophthalmology 2004;111(1):53-61.

2. Wright AF, Chakarova CF, Abd El-Aziz MM, Bhattacharya SS. Photoreceptor degeneration: genetic and mechanistic dissection of a complex trait. [Internet]. Nature reviews. Genetics 2010;11(4):273-84.

3. Ferrari $S$ et al. Retinitis pigmentosa: genes and disease mechanisms. [Internet]. Current genomics 2011;12(4):238-49.

4. Li ZY, Kljavin IJ, Milam a H. Rod photoreceptor neurite sprouting in retinitis pigmentosa. [Internet]. The Journal of neuroscience: the official journal of the Society for Neuroscience 1995;15(8):542938.

5. Byrne LC et al. Viral-mediated RdCVF and RdCVFL expression protects cone and rod photoreceptors in retinal degeneration [Internet]. Journal of Clinical Investigation 2015;125(1):105-116.

6. Punzo C, Kornacker K, Cepko CL. Stimulation of the insulin/mTOR pathway delays cone death in a mouse model of retinitis pigmentosa. [Internet]. Nature neuroscience 2009;12(1):44-52.

7. Leveillard T, Sahel JA. Rod-Derived Cone Viability Factor for Treating Blinding Diseases: From Clinic to Redox Signaling [Internet]. Science Translational Medicine 2010;2(26):26ps16-26ps16.

8. Wang SK, Xue Y, Cepko CL. Microglia modulation by TGF- $\beta 1$ protects cones in mouse models of retinal degeneration. Journal of Clinical Investigation [published online ahead of print: 2020]; doi:10.1172/JCl136160

9. Ramachandran PS, Song JY, Bennett J. Exploiting metabolic and antioxidant pathways to maintain vision in blinding disease. Journal of Clinical Investigation 2015;125(4):1390-1392.

10. Bennett J. Taking Stock of Retinal Gene Therapy: Looking Back and Moving Forward [Internet]. Molecular Therapy 2017;25(5):1076-1094.

11. Dalkara D, Duebel J, Sahel J-A. Gene therapy for the eye focus on mutation-independent approaches. [Internet]. Current opinion in neurology 2015;28(1):51-60. 
12. Sahel $\mathrm{J}$ et al. Partial recovery of visual function in a blind patient after optogenetic therapy [Internet]. Nature Medicine [published online ahead of print: May 24, 2021]; doi:10.1038/s41591-021-01351-4

13. Bi A et al. Ectopic expression of a microbial-type rhodopsin restores visual responses in mice with photoreceptor degeneration. [Internet]. Neuron 2006;50(1):23-33.

14. Chaffiol A et al. A New Promoter Allows Optogenetic Vision Restoration with Enhanced Sensitivity in Macaque Retina [Internet]. Molecular Therapy 2017;25(11):2546-2560.

15. Sengupta A et al. Red-shifted channelrhodopsin stimulation restores light responses in blind mice, macaque retina, and human retina. [Internet]. EMBO molecular medicine 2016;8(11):1248-1264.

16. Gauvain $\mathrm{G}$ et al. Optogenetic therapy: high spatiotemporal resolution and pattern discrimination compatible with vision restoration in non-human primates. Communications Biology [published online ahead of print: 2021]; doi:10.1038/s42003-020-01594-w

17. Busskamp V et al. Genetic Reactivation of Cone Photoreceptors Restores Visual Responses in Retinitis Pigmentosa [Internet]. Science 2010;329(5990):413-417.

18. Chuong AS et al. Noninvasive optical inhibition with a red-shifted microbial rhodopsin [Internet]. Nature Neuroscience 2014;17(8):1123-1129.

19. Khabou $\mathrm{H}$ et al. Noninvasive gene delivery to foveal cones for vision restoration [Internet]. $\mathrm{JCl}$ Insight 2018;3(2):1-18.

20. Sahel $\mathrm{J}$ et al. Partial recovery of visual function in a blind patient after optogenetic therapy. Nature Medicine [published online ahead of print: May 2021]; doi:10.1038/s41591-021-01351-4

21. Berry MH et al. Restoration of high-sensitivity, adapting, patterned vision with a cone opsin [Internet]. Nature Communications (2019):1-12.

22. Cehajic-kapetanovic $\mathrm{J}$ et al. Restoration of Vision with Ectopic Expression of Article Restoration of Vision with Ectopic Expression of Human Rod Opsin. Current Biology 2015;25(16):2111-2122.

23. De Silva SR et al. Long-term restoration of visual function in end-stage retinal degeneration using subretinal human melanopsin gene therapy [Internet]. Proceedings of the National Academy of Sciences 2017; 114(42):11211-11216.

24. Lin B, Koizumi A, Tanaka N, Panda S, Masland RH. Restoration of visual function in retinal degeneration mice by ectopic expression of melanopsin. [Internet]. Proceedings of the National Academy of Sciences of the United States of America 2008;105(41):16009-14.

25. van Wyk M, Pielecka-Fortuna J, Löwel S, Kleinlogel S. Restoring the ON Switch in Blind Retinas: OptomGluR6, a Next-Generation, Cell-Tailored Optogenetic Tool [Internet]. PLOS Biology 2015;13(5):e1002143.

26. Simon C-J, Sahel J-A, Duebel J, Herlitze S, Dalkara D. Opsins for vision restoration [Internet]. Biochemical and Biophysical Research Communications [published online ahead of print: January 2020];(xxxx). doi:10.1016/j.bbrc.2019.12.117

27. Masseck OA et al. Vertebrate Cone Opsins Enable Sustained and Highly Sensitive Rapid Control of G i/o Signaling in Anxiety Circuitry [Internet]. Neuron 2014;81(6):1263-1273. 
28. Lin B, Masland RH, Strettoi E. Remodeling of cone photoreceptor cells after rod degeneration in rd mice.. Experimental eye research 2009;88(3):589-99.

29. Wässle H, Grünert U, Röhrenbeck J, Boycott BB. Cortical magnification factor and the ganglion cell density of the primate retina [Internet]. Nature 1989;341(6243):643-646.

30. Whorton MR, MacKinnon R. X-ray structure of the mammalian GIRK2- $\beta$ Y G-protein complex [Internet]. Nature 2013;498(7453):190-197.

31. Hassall $\mathrm{MM}$ et al. Analysis of early cone dysfunction in an in vivo model of rod-cone dystrophy. International Journal of Molecular Sciences 2020;21(17):1-18.

32. Ye G-J et al. Cone-Specific Promoters for Gene Therapy of Achromatopsia and Other Retinal Diseases [Internet]. Human Gene Therapy 2016;27(1):72-82.

33. Greenwald DL, Cashman SM, Kumar-Singh R. Mutation-independent rescue of a novel mouse model of Retinitis Pigmentosa [Internet]. Gene Therapy 2013;20(4):425-434.

34. Duncan JL, Roorda A. Dysflective Cones [Internet]. In: Retinal Degenerative Diseases Mechanisms and Experimental Therapy. 2019:133-137

35. Berry $\mathrm{MH}$ et al. Restoration of patterned vision with an engineered photoactivatable $\mathrm{G}$ proteincoupled receptor [Internet]. Nature Communications 2017;8(1):1862.

36. Cehajic-Kapetanovic $\mathrm{J}$ et al. Restoration of Vision with Ectopic Expression of Human Rod Opsin [Internet]. Current Biology 2015;25(16):2111-2122.

37. Gaub BM, Berry MH, Holt AE, Isacoff EY, Flannery JG. Optogenetic Vision Restoration Using Rhodopsin for Enhanced Sensitivity. [Internet]. Molecular therapy: the journal of the American Society of Gene Therapy 2015;23(10):1562-71.

38. Packer AM, Roska B, Häusser M. Targeting neurons and photons for optogenetics. [Internet]. Nature neuroscience 2013;16(7):805-15.

39. Chrysostomou V, Stone J, Valter K. Life History of Cones in the Rhodopsin-Mutant P23H-3 Rat: Evidence of Long-term Survival [Internet]. Investigative Opthalmology \& Visual Science 2009;50(5):2407.

40. Baumgartner WA, Baumgartner AM. Accounting for disagreements on average cone loss rates in retinitis pigmentosa with a new kinetic model: Its relevance for clinical trials [Internet]. Medical Hypotheses 2016;89:107-114.

41. Lin B, Masland RH, Strettoi E. Remodeling of cone photoreceptor cells after rod degeneration in rd mice. [Internet]. Experimental eye research 2009;88(3):589-99.

42. Nassisi $\mathrm{M}$ et al. Near-infrared fundus autofluorescence alterations correlate with swept-source optical coherence tomography angiography findings in patients with retinitis pigmentosa [Internet]. Scientific Reports 2021;11(1):1-12.

43. Airan RD, Thompson KR, Fenno LE, Bernstein H, Deisseroth $\mathrm{K}$. Temporally precise in vivo control of intracellular signalling. [Internet]. Nature 2009;458(7241):1025-9. 
44. Schlinkmann KM et al. Critical features for biosynthesis, stability, and functionality of a G proteincoupled receptor uncovered by all-versus-all mutations. [Internet]. Proceedings of the National Academy of Sciences of the United States of America 2012;109(25):9810-5.

45. Li X et al. Fast noninvasive activation and inhibition of neural and network activity by vertebrate rhodopsin and green algae channelrhodopsin.. Proceedings of the National Academy of Sciences of the United States of America 2005;102(49):17816-17821.

46. Pennesi ME et al. Long-term characterization of retinal degeneration in rd1 and rd10 mice using spectral domain optical coherence tomography.. Investigative ophthalmology \& visual science 2012;53(8):4644-56.

\section{Figures}

A G-proteir-coupled

receptor (GPCR)
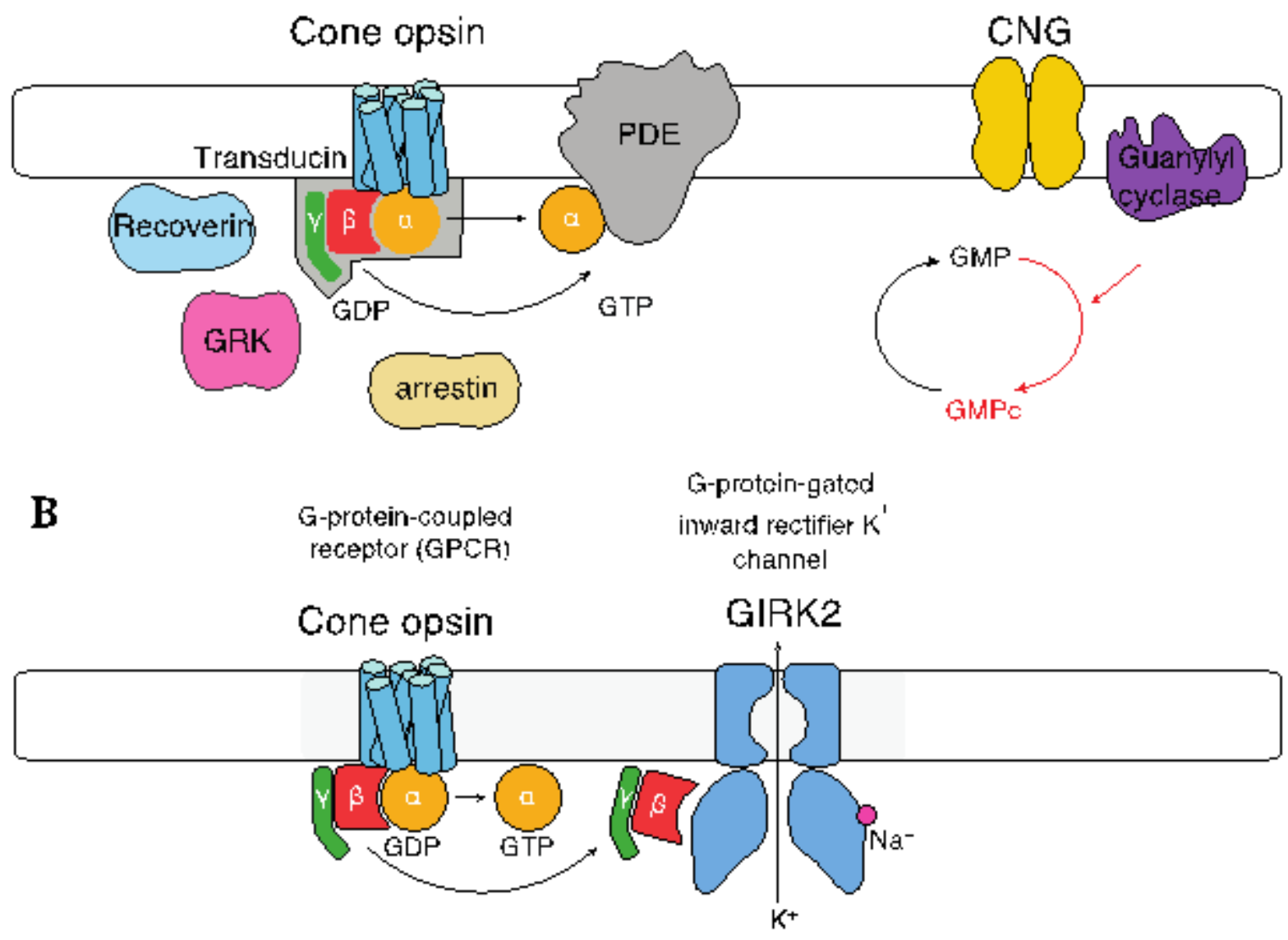

Figure 1

Phototransduction cascade in the mammalian cone photoreceptor.

(A) Phototransduction cascade proteins in cones. The absorption of a photon activates the cone opsin. Light absorption activates the opsin which becomes catalytically active. The $\mathrm{G}$ protein, transducin, 
transduces the signal via its a-subunit activated by the replacement of GDP by GTP. Then, the a-subunit dissociates from the $\beta y$ subunits to activate the membrane-associated phosphodiesterase (PDE6C) by removing its two regulatory $(\gamma)$ subunits. The activated phosphodiesterase hydrolyses cGMP to GMP. The reduction of cGMP closes the channel, and this stops cation entry resulting in net hyperpolarization. After activation, the phototransduction cascade is deactivated by two mechanisms: (i) the transducin inactivates itself by hydrolyzing the bound GTP and (ii) the rhodopsin kinase phosphorylates the opsin that interacts with the regulatory protein arrestin, leading to opsin inactivation. After inactivation, the cone photoreceptor can be reactivated by another light stimulus. (B) Short phototransduction cascade proposed in this study. The cascade involves endogenous cone opsin and ectopically expressed GIRK2. The endogenous cone opsin is activated by light absorption recruiting an endogenous $G$ protein. The $\beta y$ subunits of this $\mathrm{G}$ protein activate the GIRK2 resulting in the efflux of $\mathrm{K}^{+}$ions leading to a hyperpolarization. Pde: Phosphodiesterase. CNG: Cyclic-nucleotide gated channels. cGMP: cyclic guanosine monophosphate. Adapted from (30)

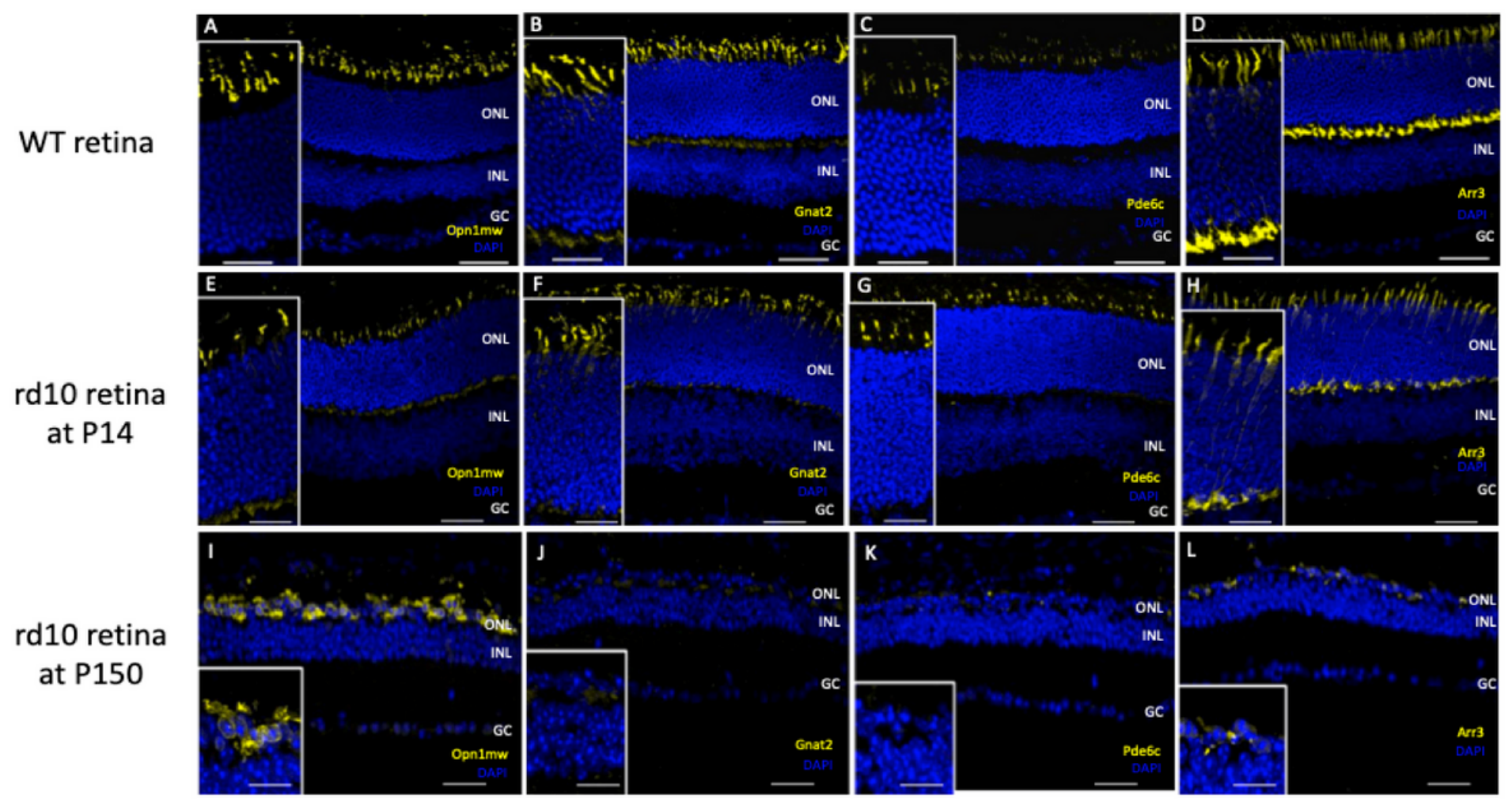

Figure 2

Immunohistochemistry analysis of cone phototransduction cascade proteins in the rd10 mouse model for rod-cone dystrophy. (A-D) Retinal cross-section of a control WT mouse stained with DAPI (b/ue) (A) opsin (Opn1mw) (yellow), (B) transducin (Gnat2) (yellow), (C) phosphodiesterase (Pde6c) (yellow) and (D) cone arrestin (Arr3) (yellow). (E-H) Retinal cross-section of a rd10 mouse at P14 stained with DAPI (blue) (E) opsin (Opn1mw) (yellow), (F) transducin (Gnat2) (yellow), (G) phosphodiesterase (Pde6c) (yellow) and $(\mathrm{H})$ cone arrestin (Arr3) (yellow). (I-L) Retinal cross-section of a rd10 mouse at P150 stained 
with DAPI (blue) (I) opsin (Opn1mw) (yellow), (J) transducin (Gnat2) (yellow), (K) phosphodiesterase (Pde6c) (yellow) and (L) cone arrestin (Arr3) (yellow).

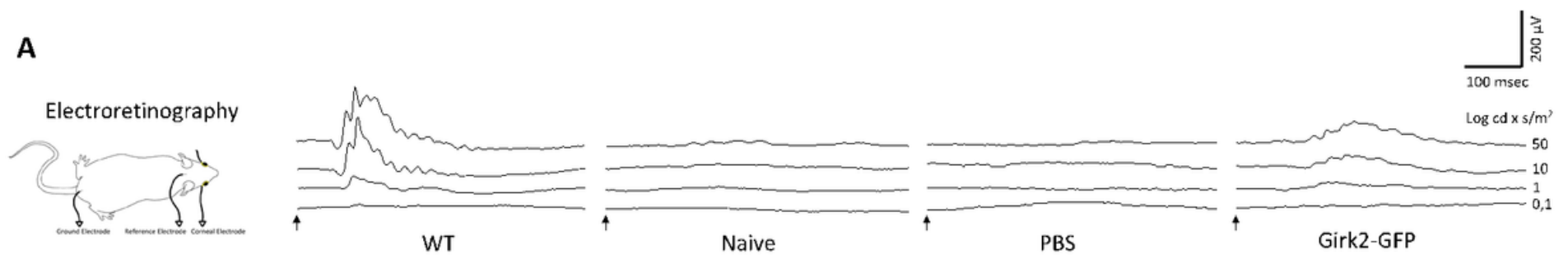

B

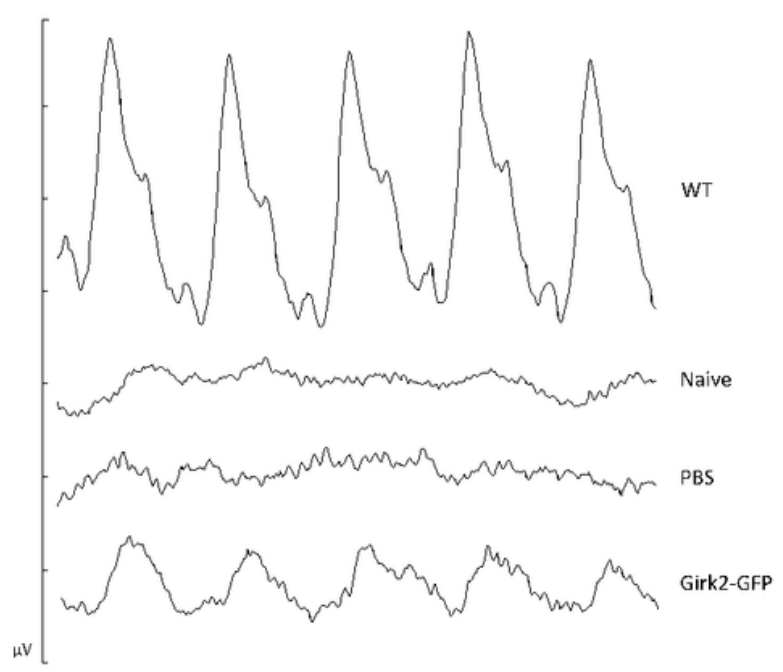

D

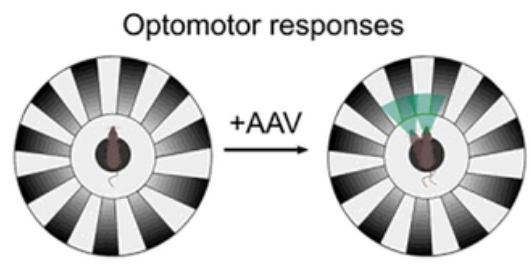

C
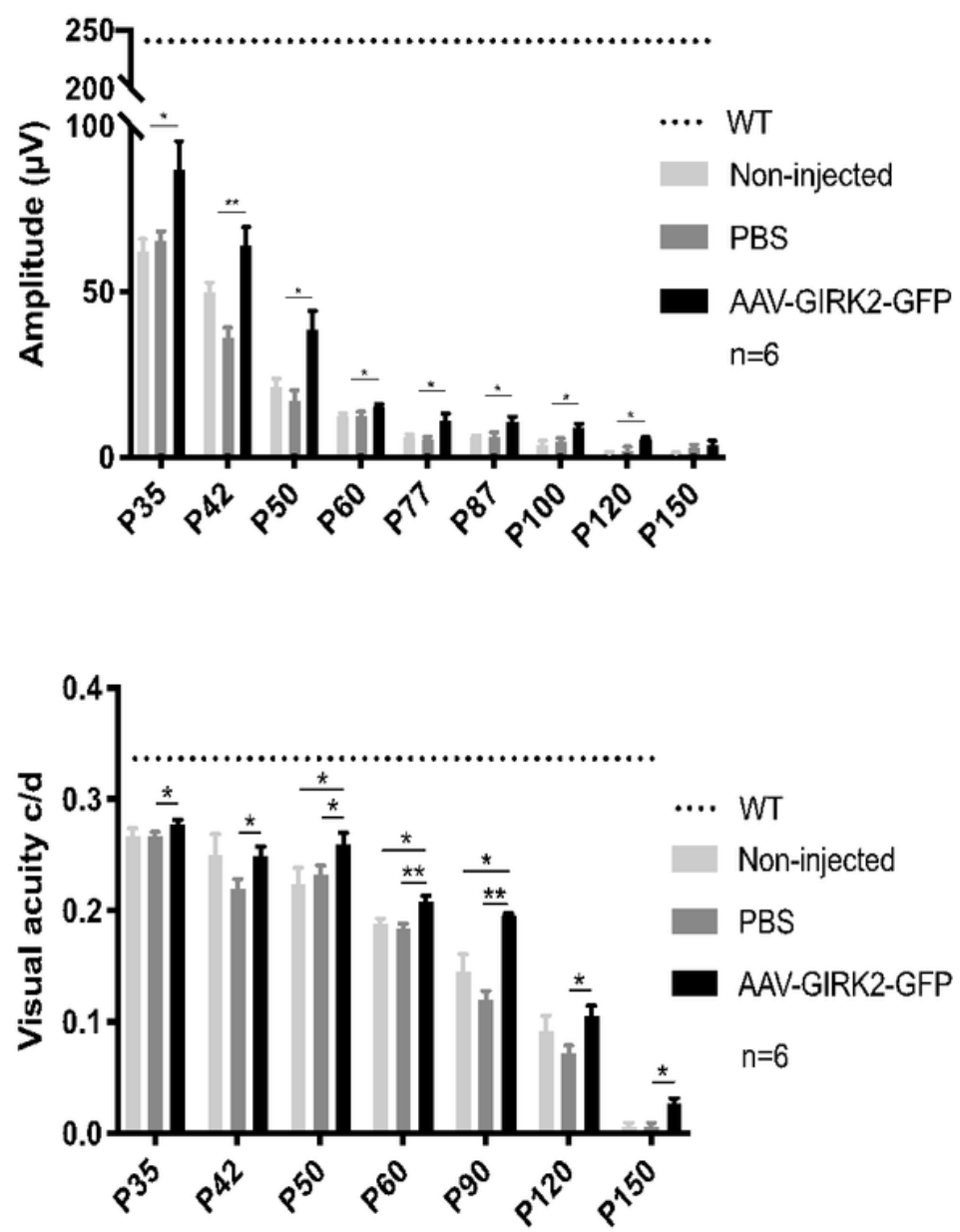

Figure 3

Long-term effect of GIRK2 gene transfer on visual responses of rd10 mice. (A) Representative photopic bwave traces recorded from $r d 10$ mice injected with AAV-GIRK2-GFP compared with those injected with PBS-pluronic $0,001 \%$ or non-injected naive rd10 mice at P50. (B) Representative flicker ERGs recorded from wild-type (WT) mice and rd10 mice injected with AAV-GIRK2-GFP, PBS-pluronic 0,001\% or noninjected naive $r d 10$ mice at P50. (C) Photopic ERG amplitudes (50 cd.s $\left./ \mathrm{m}^{2}\right)$ in $r d 10$ mice injected with 
AAV-Girk2-GFP. Control eyes were injected with PBS-pluronic 0,001\% $(n=6)$. (D) Measure of the visual acuity by optokinetic reflex in $r d 10$ mice injected with AAV-GIRK2-GFP. Control eyes were injected with PBS-pluronic $0.001 \%(n=6)$.

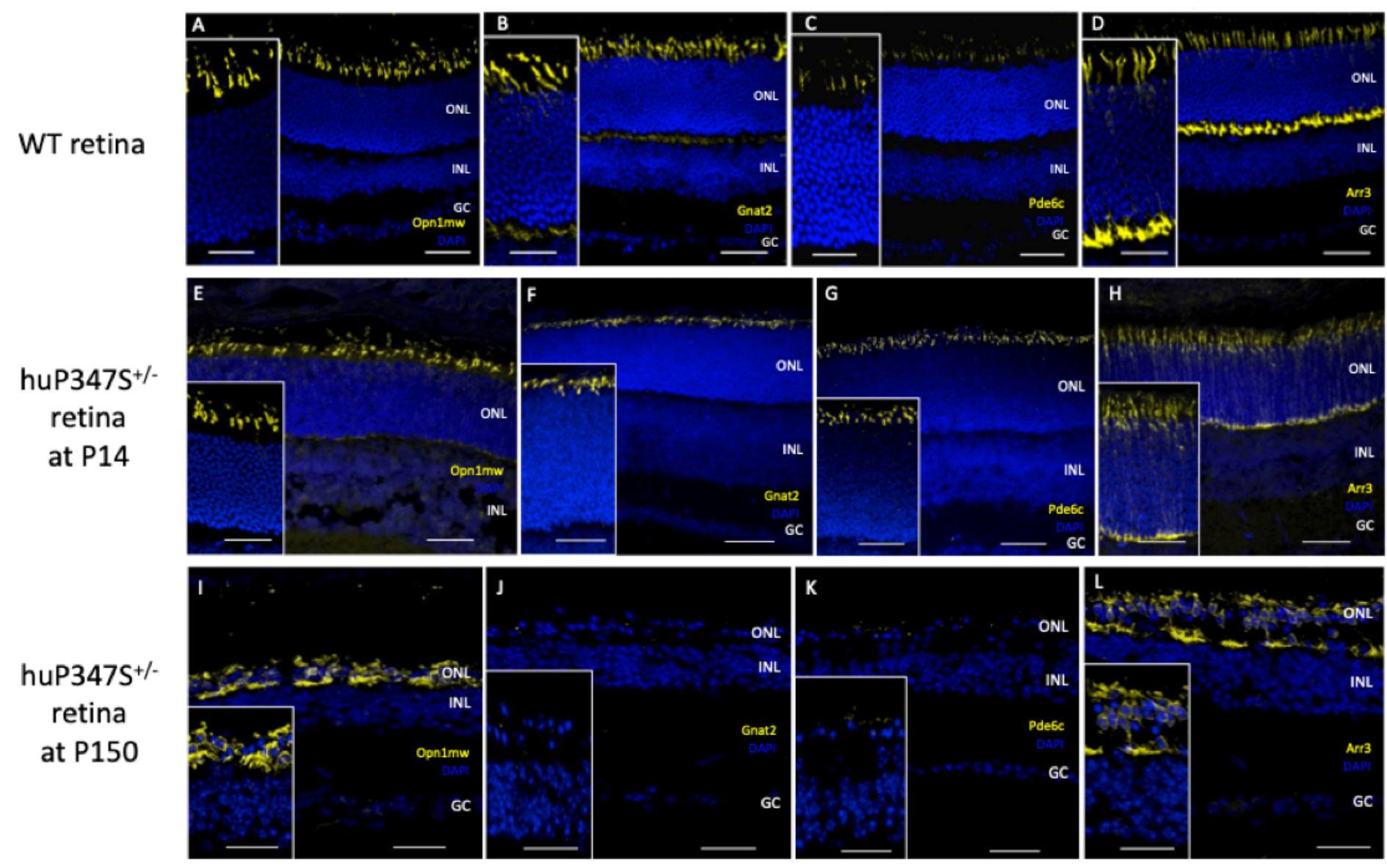

\section{Figure 4}

Immunohistochemistry against phototransduction cascade proteins in huP347S ${ }^{+/-}$mice at P150 (A-D) Retinal cross-section of a control WT mouse stained with DAPI (blue), (A) opsin (Opn1mw) (yellow), (B) transducin (Gnat2) (yellow), (C) phosphodiesterase (Pde6c) (yellow) and (D) cone arrestin (Arr3) (yellow). (E-H) Retinal cross-section of a huP3475 ${ }^{+-}$mouse at P14 stained with DAPI (blue), (E) opsin (Opn1 mw) (yellow), (F) transducin (Gnat2) (yellow), (G) phosphodiesterase (Pde6c) (yellow) and $(\mathrm{H})$ cone arrestin (Arr3) (yellow). (I-L) Retinal cross-section of a huP3475 ${ }^{+/-}$mouse at P150 stained with DAPI (blue), (I) opsin (Opn1mw) (yellow), (J) transducin (Gnat2) (yellow), (K) phosphodiesterase (Pde6c) and (L) cone arrestin (Arr3) (yellow). ONL: outer nuclear layer. INL: inner nuclear layer. GC: ganglion cells. Scale bar is $50 \mu \mathrm{m}$. Inset scale bar is $25 \mu \mathrm{m}$. 
A

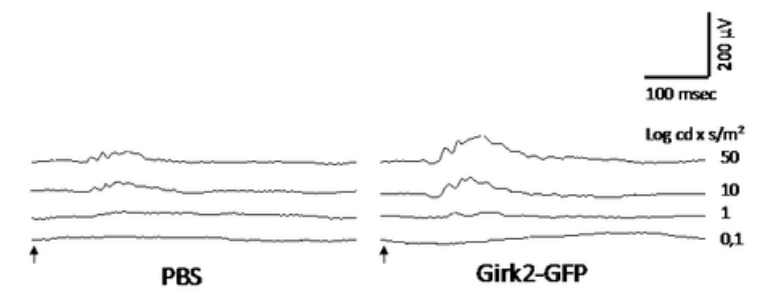

B

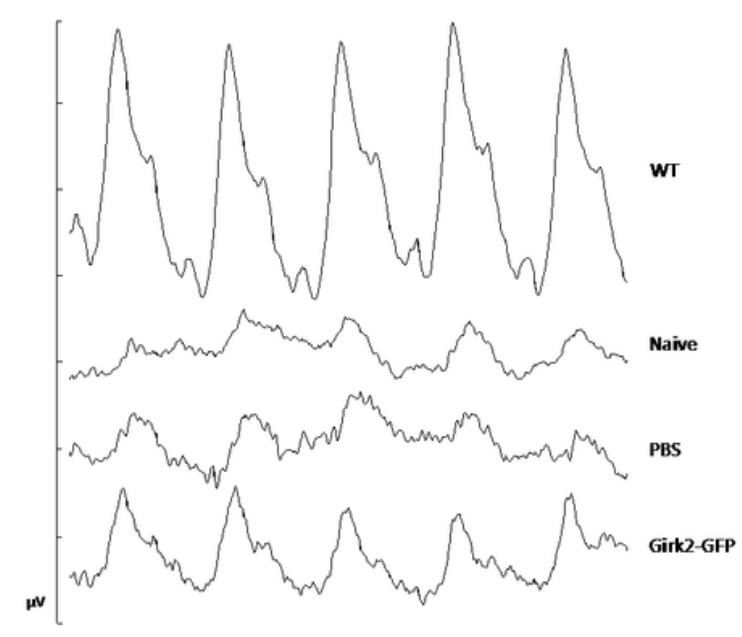

D

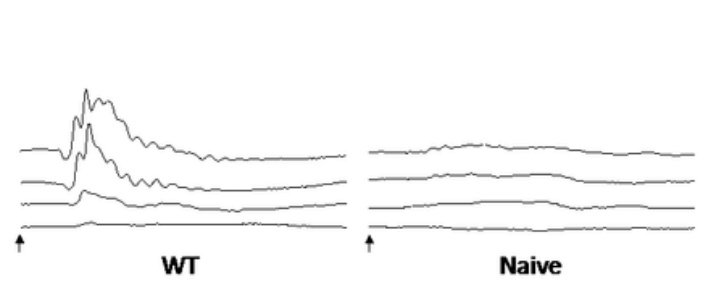

Naive

PBS

C
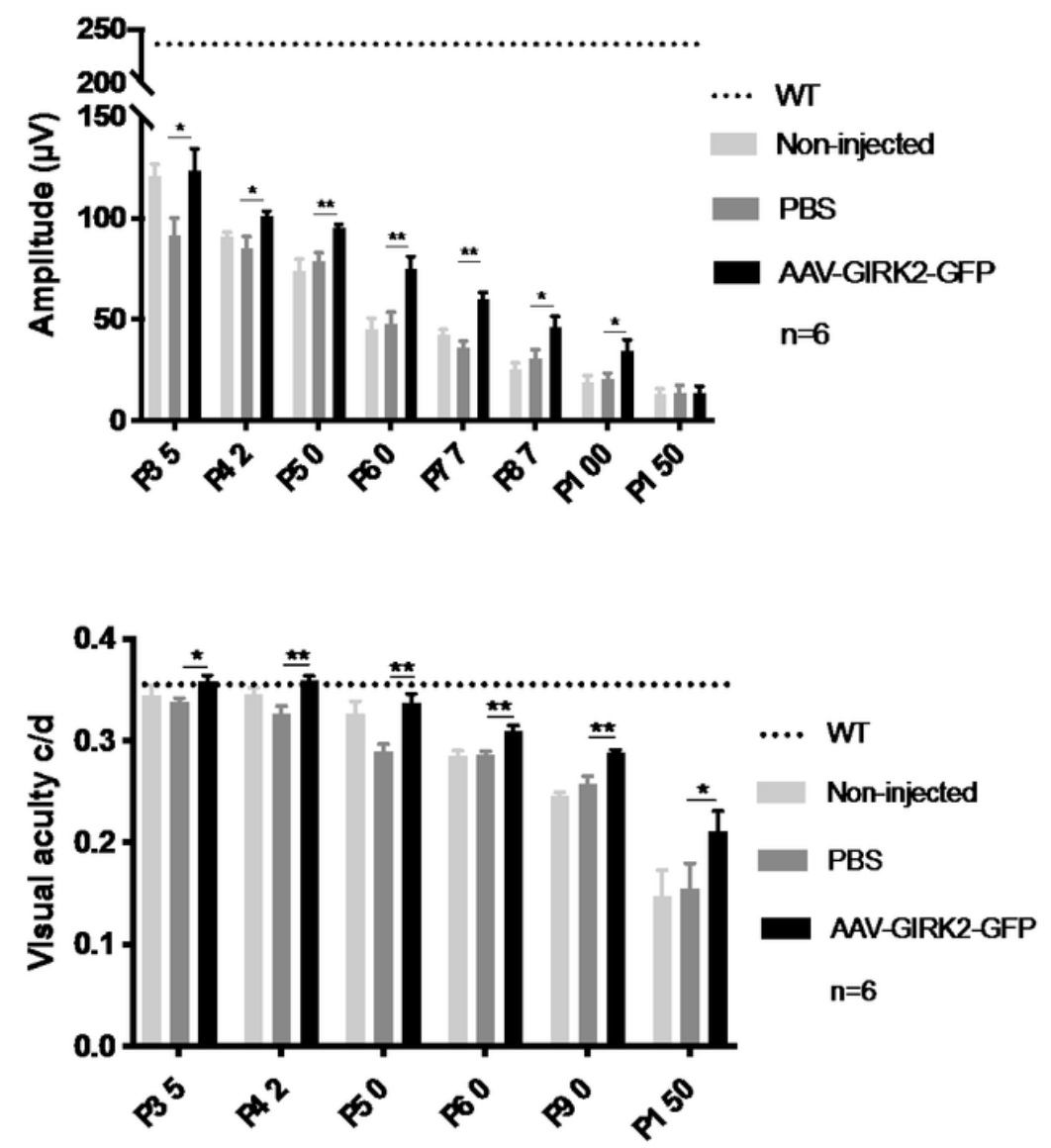

Figure 5

Effect of GIRK2 expression on retinal function of huP347S ${ }^{+/-}$mice. (A) Photopic b-wave amplitude traces were significantly higher in huP347S ${ }^{+/-}$mice injected with AAV-GIRK2-GFP compared with those injected with PBS-pluronic 0,001\% or non-injected naive huP347S ${ }^{+/-}$mice at P60. (B) Representative flicker ERGs recorded from wild-type (WT) mice and $h u P 347 S^{+/-}$mice injected with AAV-GIRK2-GFP, PBS-pluronic 0,001\% or non-injected naive huP347S ${ }^{/-}$mice at P60. (C) Photopic ERG amplitudes (50 cd.s/ $\left.\mathrm{m}^{2}\right)$ in huP347S ${ }^{+/-}$mice injected with AAV-Girk2-GFP. Control eyes were injected with PBS-pluronic 0,001\% $(n=6)$. (D) Measure of the visual acuity by optokinetic reflex in huP347S ${ }^{+-}$mice injected with AAV-GIRK2-GFP. Control eyes were injected with PBS-pluronic 0.001\% $(n=6)$. 

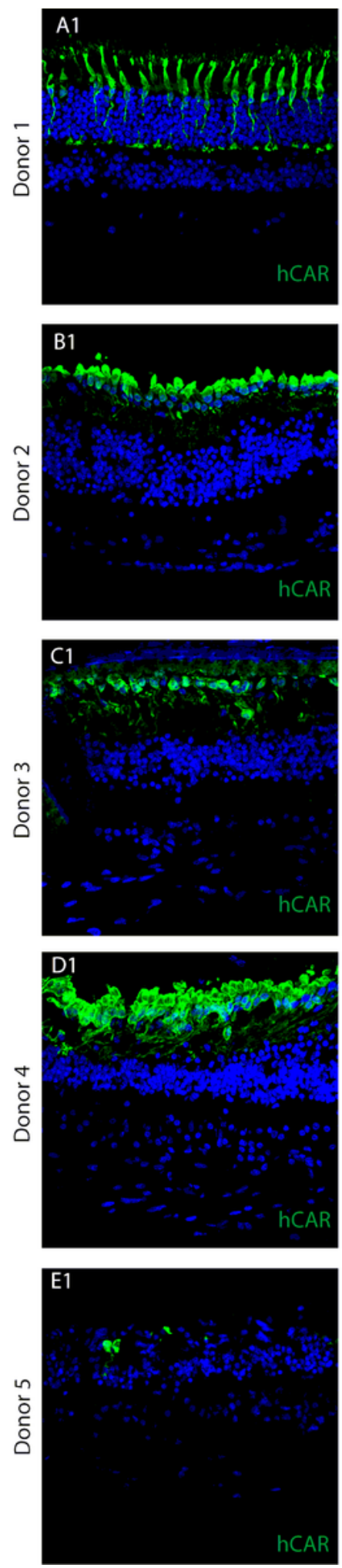
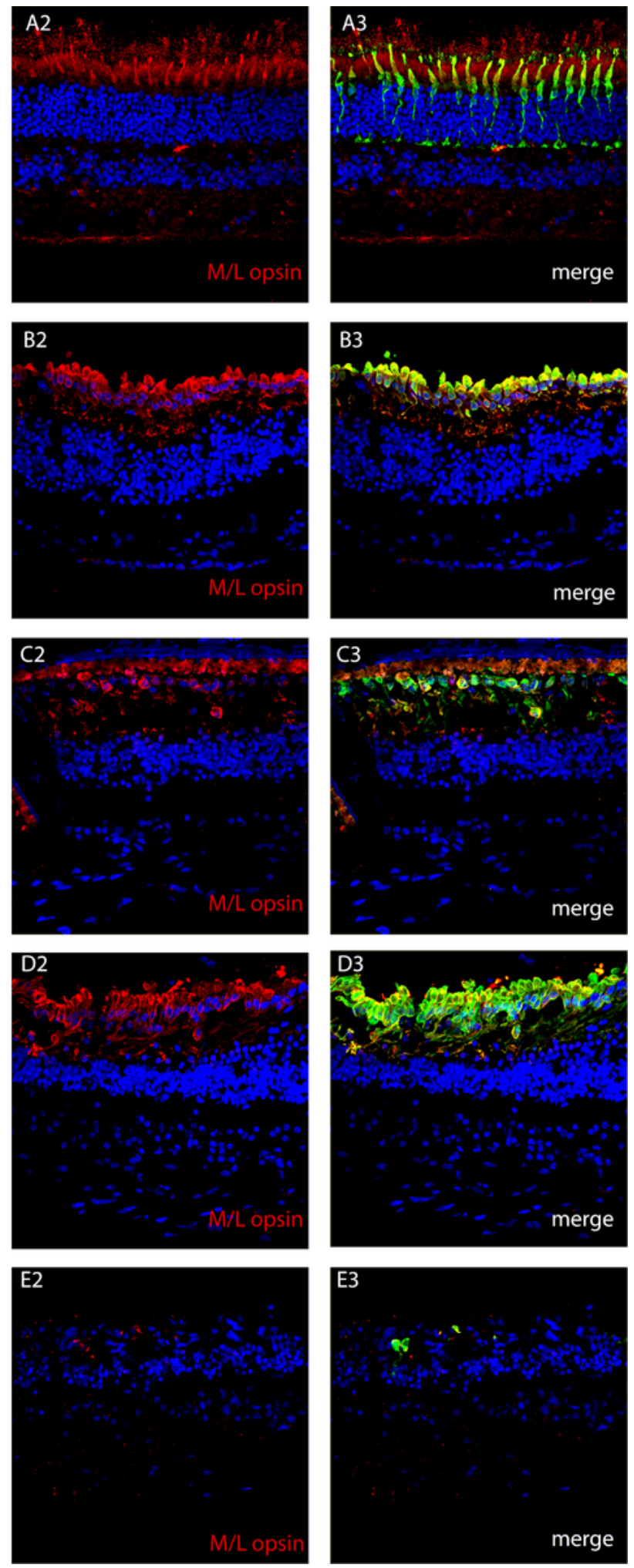

Figure 6

Cone opsin and arrestin expression in normal and RCD human retinal tissue.

Human cone arrestin (Arr3) and M/L opsin immunostainings are shown in green and red, respectively. Nuclei are stained with DAPI, in blue. (A) Retinal cross-section of a 91-year-old individual with no visual impairment (40x). (B-E) Retinal cross-sections of human RCD maculae from 4 different donors (40x). 

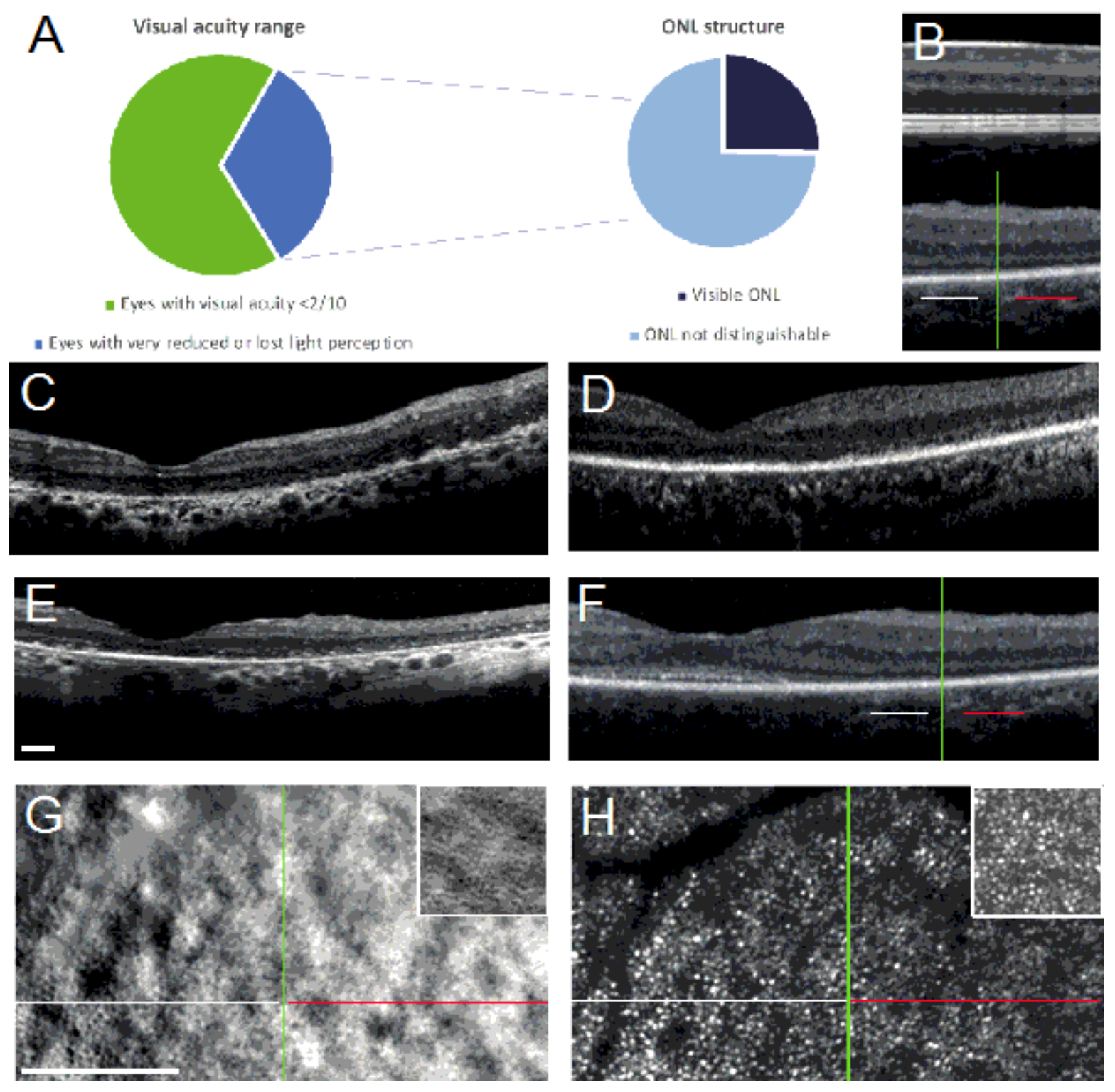

Figure 7

Phenotyping RP patients to define an eligible population for GIRK2 gene therapy.

(A) Proportion of retinitis pigmentosa patients with very reduced or lost light perception and have a detectable ONL filled with diminished outer-segment cone photoreceptor cells. (B) Zooms on OCT scans of a healthy retina (top) versus the patient shown in ( $F-H)$ suffering from retinitis pigmentosa (bottom). The green line on the OCT cross section marks the transition between a zone with external limiting membrane, implying some residual outer segment structure (white bar) and a zone with absent external limiting membrane (red bar). (C-F) Representative OCT scans from the left eye of four RP patients (aged from 32 to 77 years old) suffering from retinitis pigmentosa. (G-H) AOSLO images over the same zone shown in (B) on a retinitis pigmentosa patient, with inserts from a healthy subject for comparison. Split detection $(\mathbf{G})$ and confocal $(\mathbf{H})$ modalities show transition from clear cone mosaics (white 
bar) suggesting cones with some residual structure maintaining them in a mosaic packing, to presumed damaged cones with no clear mosaic visible (red bar) over this region. Scale bar, $200 \mu \mathrm{m}$.

\section{Supplementary Files}

This is a list of supplementary files associated with this preprint. Click to download.

- Supplementaryinformation.docx 